\title{
Fast multiplane functional imaging combining acousto-optic switching and remote focusing
}

Ee Zhuan Chong, Inês Barreiros, Bei Li, Michael M. Kohl, Martin J. Booth

Ee Zhuan Chong, Inês Barreiros, Bei Li, Michael M. Kohl, Martin J. Booth, "Fast multiplane functional imaging combining acousto-optic switching and remote focusing," Proc. SPIE 10070, Three-Dimensional and

Multidimensional Microscopy: Image Acquisition and Processing XXIV, 100700X (17 February 2017); doi: 10.1117/12.2251476

SPIE. Event: SPIE BiOS, 2017, San Francisco, California, United States 


\title{
Fast Multiplane Functional Imaging Combining Acousto-Optic Switching and Remote Focusing
}

\author{
Ee Zhuan Chong ${ }^{\mathrm{a}}$, Inês Barreiros ${ }^{\mathrm{b}}$, Bei Li ${ }^{\mathrm{a}}$, Michael M. Kohl ${ }^{\mathrm{b}}$, Martin J. Booth ${ }^{\mathrm{a}}$ \\ ${ }^{a}$ Department of Engineering Science, University of Oxford, Parks Road, Oxford, OX1 3PJ, United \\ Kingdom \\ ${ }^{b}$ Department of Physiology, Anatomy and Genetics, University of Oxford, South Parks Road, OX1 \\ 3QX, United Kingdom \\ *Corresponding author: martin.booth@eng.ox.ac.uk
}

\begin{abstract}
Networks of neurons are inherently three-dimensional in nature, whereas conventional imaging methods, such as laser scanning two-photon microscopy, usually provide only fast two-dimensional imaging. Rapid volumetric imaging would however be preferable for imaging neurons. To get a more complete picture of the dynamics of the neuron-to-neuron interactions, we have developed a pseudo-parallelised multi-plane two-photon excitation imaging system through the incorporation of an acousto-optic switching and a remote focusing technique into a resonant scanning microscope. This permits the recording of millisecond scale fluorescence transients of calcium indicators from large populations of neurons upon neural firing events at multiple chosen axial planes in very short time frame. While the remote focusing system offers aberration-free axial scanning over a few hundreds of micrometres of depth, the acousto-optic deflector provides high speed optical switching between different laser beam paths in sub-microsecond timescale which in turn, controls the axial focal plane to be targeted. Here, we report on the development of the high temporal resolution multi-plane targeted microscope and its potential application.
\end{abstract}

Keywords: Two-photon excitation, acousto-optic deflector, remote focusing, neuron signalling, multiplane imaging, fluorescence.

\section{INTRODUCTION}

Two-photon (2P) excitation fluorescence imaging is a useful tool to study biological samples at the cellular level with applications not only restricted to two-dimensional (2D) but also three-dimensional (3D) specimens. Using 2P excitation, the generated fluorescence signal becomes quadratic dependent on the excitation intensity. Because of this nonlinear nature of fluorophore excitation, the use of short-pulsed lasers and a tightly focused high intensity laser spot with a high numerical aperture (NA) objective lenses provides optical sectioning without the need of sub-micron pinhole in the detection path as typically present in a confocal microscope. In the early 1990s, Denk, W. et al were among the first to demonstrate the application of 2P laser scanning imaging technique on biological specimens and showcase its advantages over the conventional confocal laser scanning method ${ }^{1}$. For instance, the pinhole throughput loss was avoided and a reduced outof-focus photobleaching effect on samples was observed due to the highly localised excitation. Thus, there is a growing interest within the neuroscience research community to use the noninvasive $2 \mathrm{P}$ imaging technique to study the complex brain structure and the neuron activities in small vertebrates and non-vertebrates. Due to the excitation wavelength required for 2P imaging, the wavelength generally falls within the near infrared (NIR) spectral window. Thus, the penetration depth can be extended further into the tissue sample compared to that of one photon (1P) excitation counterpart owing to the lower scattering coefficient experienced by the laser beam path traveling through the turbid medium ${ }^{2,3}$. With this longer excitation wavelength, the benefit of the greater focusing depth for the $2 \mathrm{P}$ microscope becomes apparent when imaging neuron population lying within layers deep inside the brain. Kobat, D. et al demonstrated that deep tissue in vivo imaging on mouse brain is possible with the use of femtosecond pulsed laser with the wavelength set at $1280 \mathrm{~nm}^{4}$. A $1.6 \mathrm{~mm}$ penetration depth was achieved enabling the capturing of the images of cortical vasculature in an intact mouse brain. However, the speed of the raster scanning technique that builds the 2D image can become critical when performing 3D volume sweep across a millimeter thick biological specimen. It dictates the frame rate of the $2 \mathrm{P}$ microscopy system. To generate an image of $512 \times 512$ pixels, the frame rate achievable for $2 \mathrm{D}$ galvanometer scanner is generally limited to 4 frames per second (fps). Therefore, a range of ingenious imaging solutions have been devised to push the frame rate limit

Three-Dimensional and Multidimensional Microscopy: Image Acquisition and Processing XXIV, edited by

Thomas G. Brown, Carol J. Cogswell, Tony Wilson, Proc. of SPIE Vol. 10070, 100700X

(c) 2017 SPIE · CCC code: 1605-7422/17/\$18 - doi: 10.1117/12.2251476

Proc. of SPIE Vol. $10070100700 X-1$ 
higher, e.g. polygonal scan mirror ${ }^{5}$, light sheet illumination ${ }^{6}$, parallerised multifocal scanning ${ }^{7}$ and spatio-temporal focusing of excitation ${ }^{8}$ to name a few. Alternatively, a more straightforward approach to improve the $2 \mathrm{P}$ imaging speed is to replace the $\mathrm{x}$-scanning galvanometer mirror with a resonant mirror which vibrates at a high frequency (typically at 8 $\mathrm{kHz}$ ). This enables an imaging speed reaching a video rate around $30 \mathrm{fps}$. For instance, though at a slightly lower image size of $500 \times 450$ pixels, Nguyen, Q. et al have demonstrated the development of the early version of custom built $2 \mathrm{P}$ resonant scanning system that could perform video-rate calcium imaging that was fast enough to detect the $\mathrm{Ca}^{2+}$ transients in the dendrites and spines of cortical neurons ${ }^{9}$. Following technological advancement, now resonant scanning microscopy systems are widely available commercially. However, to record neural activity over large volume, some challenges are still posed. Unlike the volumetric structural imaging, simple translation of the objective along the z-direction is not sufficiently fast to match the millisecond temporal response of neurons in 3D volume. Therefore, with the purpose of enhancing the volume imaging speed, we modified an existing commercial $2 \mathrm{P}$ resonant microscope by incorporating an acousto-optic deflector (AOD) and a remote focusing system ${ }^{10}$. The former addition provides the fast optical switching capability, while the latter offers aberration-free axial displacement of focal spot with both the objective and the specimen kept immobile. Hence, by combining the resonant scanner, the AOD and the remote focusing, we developed a fast multiplane imaging system that can be useful to study the neuron-to-neuron interactions between widely separated layers in a brain.

\section{MATERIALS AND METHODS}

The multiplane functional imaging capability was built around an existing Sutter MOM 2P resonant system and the schematic of the optical setup is illustrated in Fig. 1.

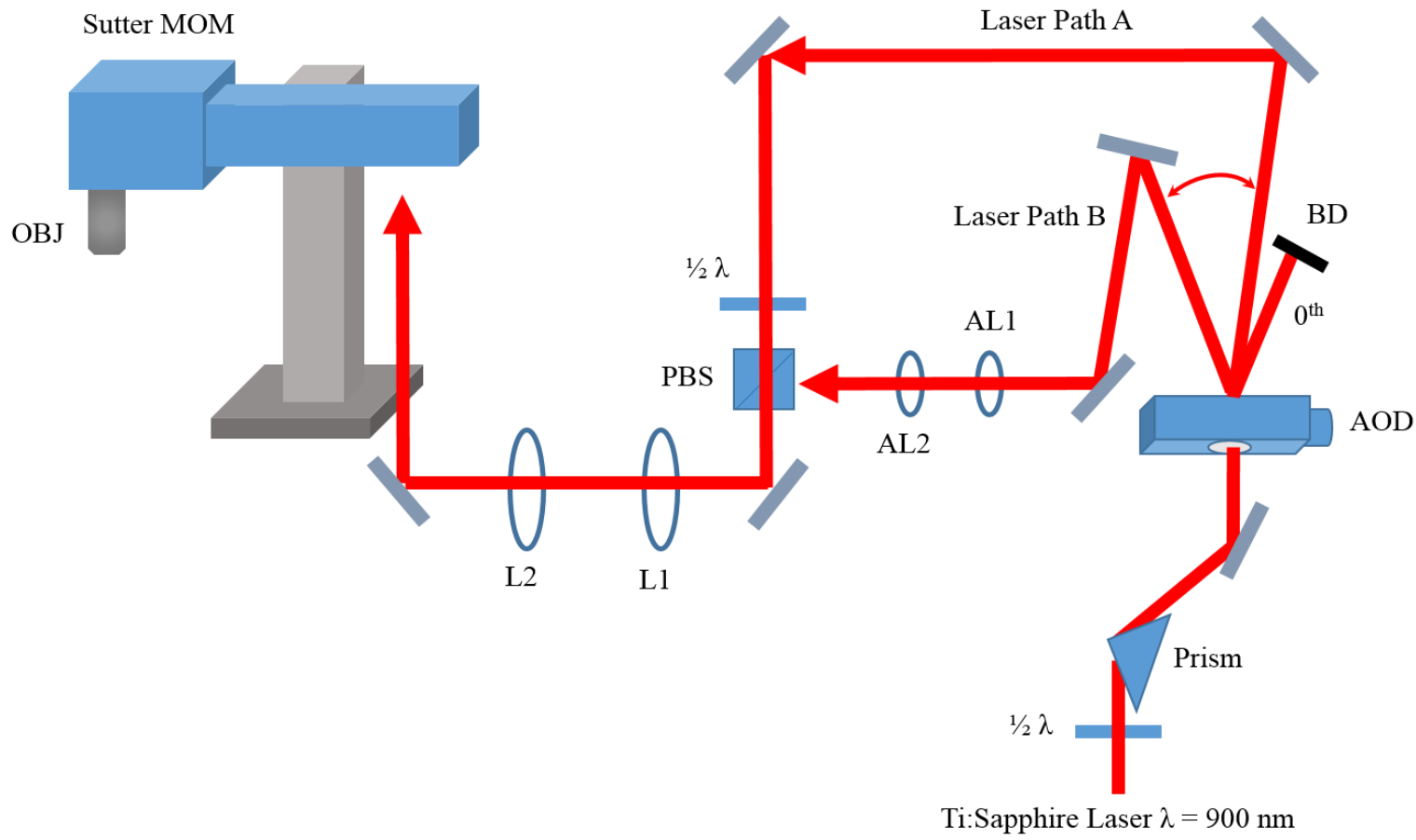

Figure 1. Simplified schematic diagram of the fast multiplane 2P microscopy system depicting two respective first-order diffracted laser paths, Path A and Path B. Path A is in-focus beam path and Path B is remote focused beam path. $1 / 2 \lambda$ : half-wave plate; AOD: acousto-optic deflector; BD: beam dump; AL1, AL2: aspheric lenses; PBS: polarising beamsplitter; L1, L2: lenses; OBJ: objective. 
An AOD can offer microsecond beam steering performance. Depending on the applied frequency of the acoustic wave, the angle of beam deflection can be controlled based on ${ }^{11}$

$$
\theta=\frac{\lambda f}{V}
$$

where $\lambda$ is the incident laser wavelength, $f$ is the frequency of the acoustic wave and $V$ is the velocity of the acoustic wave which is typically constant for certain dispersive material used. For example, the velocity of the acoustic wave travelling across tellurium dioxide $\left(\mathrm{TeO}_{2}\right)$ crystal is $\sim 650 \mathrm{~ms}^{-1}$. Here, the AOD (DTSX-400-980, AA Optoelectronic) was used as the fast microsecond optical switch that deflected the incoming laser beam into two separate paths and were later recombined by a PBS as shown in Fig. 1. Because AOD is a highly dispersive element, an ultrafast laser will experience some spatial and temporal broadening when propagating through the device. These distortions can reduce the acquired image quality greatly in terms of the signal-to-noise ratio (SNR) as well as the resolution. Therefore, an uncoated SF11 equilateral prism (PS859, Thorlabs) was paired with the AOD to compensate for the spatial and temporal dispersion induced in the AOD. To achieve an optimal spatial and temporal dispersion compensation for a Ti:Sapphire laser (Mai Tai, Spectra-Physics) with the wavelength tuned to $900 \mathrm{~nm}$, the prism was positioned such that the laser incident angle to the prism is $\sim 56^{\circ}$ and the prism-AOD separation is $\sim 25 \mathrm{~cm}$. The spatio-temporal restoration was performed based on the centre scan frequency of the AOD at $900 \mathrm{~nm}$ wavelength i.e. $87 \mathrm{MHz}$. The detailed work and analyses on the prism-AOD dispersion compensation were previously reported by Zeng, $\mathrm{S}$ et $a l^{12,13}$. The resultant laser beam profile after the spatial dispersion compensation was imaged onto an exposed CCD of a webcam (LifeCam, Microsoft) and the final recovered laser pulsewidth after the temporal dispersion compensation was measured using an interferometric autocorrelator (pulseCheck, APE). Apart from the AOD as the fast optical switch, the remote focusing technique affords the distinct flexibility to displace the focal spot aberration-free over tens to hundreds of micrometers deep into biological tissues with no sample or objective movement required ${ }^{10,14}$. A remote focusing system requires high numerical aperture lenses to provide the correct, aberration-free focusing ${ }^{10}$. Considering the cost of implementation, instead of objective lenses, a pair of identical 0.68 NA aspheric lenses (C330TMD-B, Thorlabs) as depicted in Fig. 1 was utilised to perform the remote focusing. The aspheric lens with the NA of 0.68 was chosen in order to match the acceptance angle of the final excitation objective. While one of the aspheric lenses was fixed on a cage plate, the other was mounted on a multi-axis translation stage (MBT616D/M, Thorlabs). The back pupil plane of the aspheric lens was reimaged onto the back pupil plane of the 0.8 NA $16 \times$ water dipping objective (Nikon) by a series of $4 \mathrm{f}$ systems. A pollen grains slide (Griffin Education) was chosen as the preliminary test sample for the volumetric multiplane imaging.

\section{RESULTS AND DISCUSSION}

The laser beam spot after the prism-AOD configuration was imaged onto the webcam CCD and the images acquired are shown as below: 
(a)

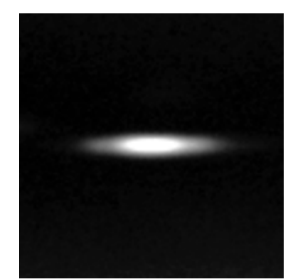

(b)

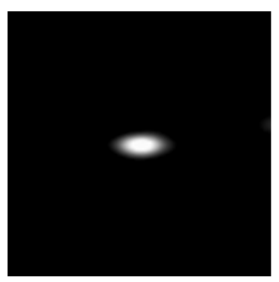

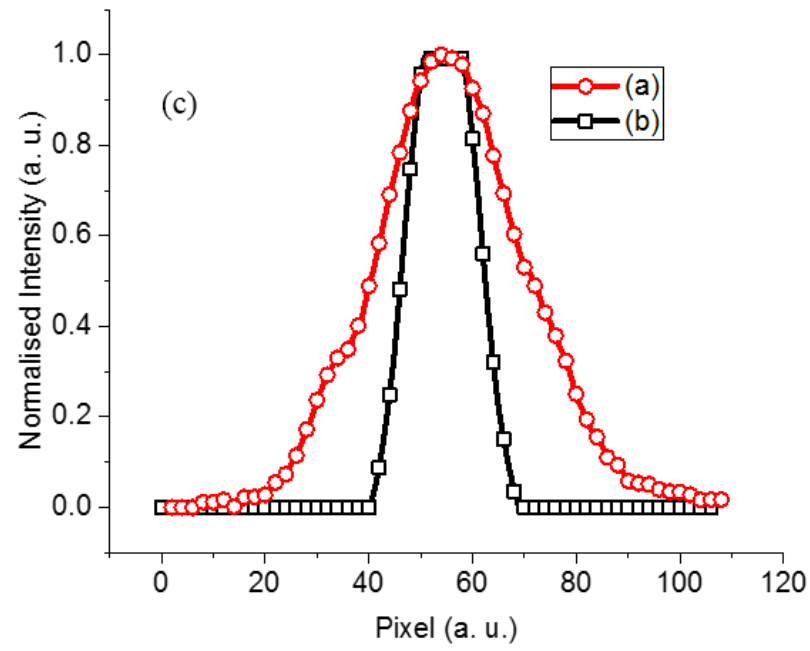

Figure 2. Laser beam profiles after the first-order diffraction by the AOD. (a) Beam spot without the presence of the compensating prism. (b) Beam spot after the spatial dispersion compensation by the prism-AOD pair. (c) Horizontal line profiles across the beam spots in uncompensated (a) and compensated (b) images.

Fig. 2 shows a marked reduction in the beam broadening, by a factor of $\sim 2$, along the $\mathrm{x}$-axis when the spatial dispersion induced by the AOD was correctly counter-balanced by the material dispersion of the prism and the angle of laser incidence on the prism. To compensate for the temporal dispersion, the distance between the prism and the AOD was adjusted to $\sim 25 \mathrm{~cm}$ such that the minimum pulsewidth of the Ti:Sapphire laser was achieved. The change of the pulsewidth with increasing prism-AOD distance was measured with the autocorrelator, as plotted in Fig. 3. 

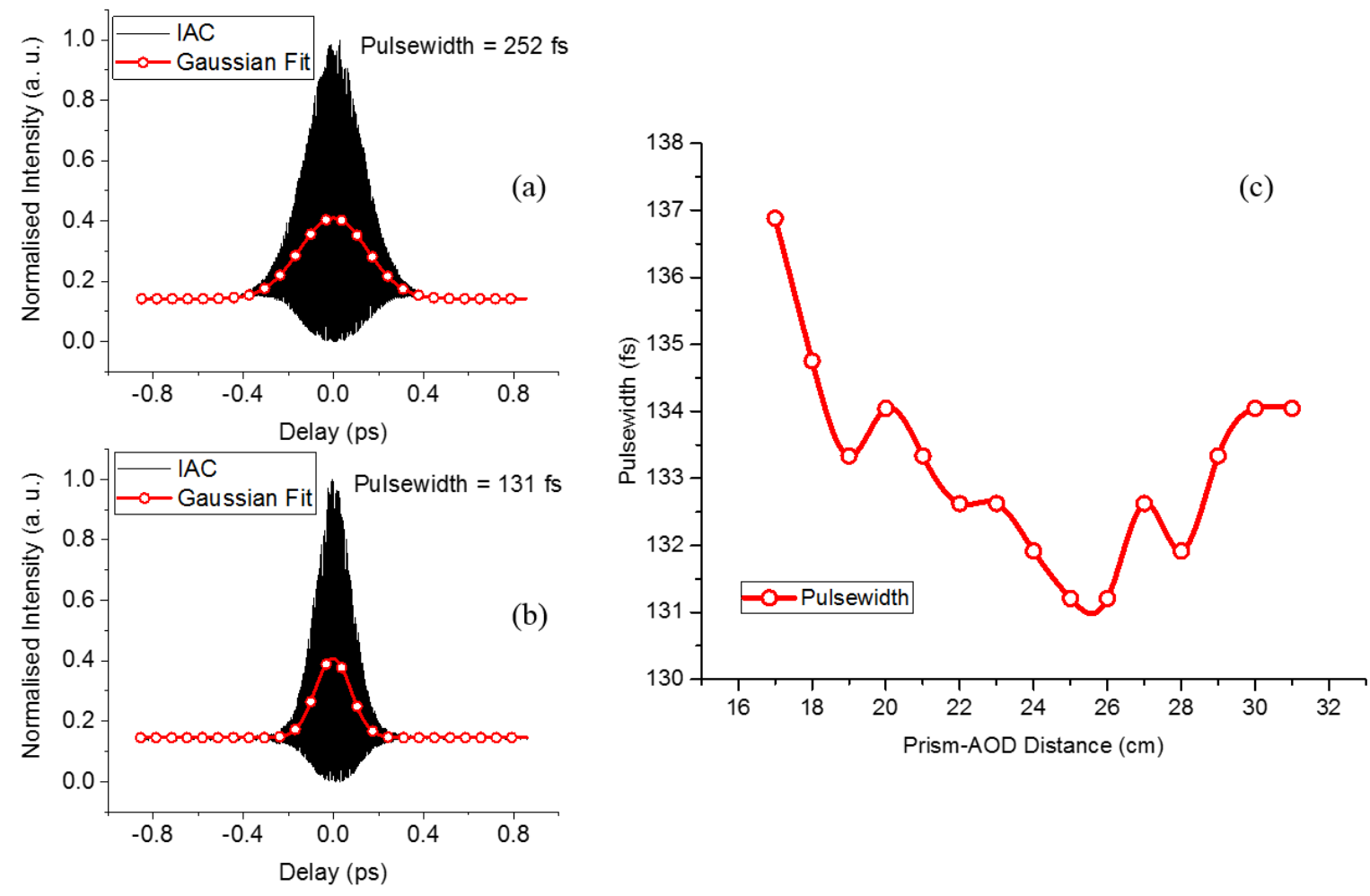

Figure 3. Pulsewidth measurements of femtosecond Ti:Sapphire laser using an interferometric autocorrelator. (a)

Empirical interferometric autocorrelation (IAC) trace alongside the corresponding Gaussian fit for the first-order diffracted beam without temporal dispersion compensation. (b) IAC trace of the ultrafast laser and the corresponding Gaussian fit after the insertion of prism $25 \mathrm{~cm}$ apart from the AOD to compensate for the AOD induced dispersion. (c)

The change of the measured laser pulsewidth as a function of the prism-AOD distance.

As indicated in Fig. 3(a) and Fig. 3(b), the incorporation of SF11 prism with an apex angle of $60^{\circ}$ preceding the AOD was able to compensate for the temporal dispersion in the AOD which in turn, resulted in the significant recovery of the laser pulsewidth from $\sim 250$ fs to $\sim 130 \mathrm{fs}$ when the prism-AOD distance is $\sim 25 \mathrm{~cm}$. From Fig. 3 (c), a reasonable temporal dispersion compensation can be realised with the prism-AOD separation falls within 24.5-26.5 cm range.

To demonstrate the functionality of the multiplane $2 \mathrm{P}$ microscope with compensation of the spatio-temporal dispersion of AOD and remote focusing, we acquired some autofluorescence images of pollen grains at different focal depths by manually adjusting the aspheric lens's position along the optical axis. The relative focal shift introduced by the axial translation of the aspheric lens can be estimated based on ${ }^{10}$

$$
Z=\left(\frac{n_{2}}{n_{1}}\right) Z_{R}
$$

where $z$ is the resulting axial shift of the excitation focal plane, $n_{2}, n_{l}$ are the refractive indices of the immersion media at the remote focusing and the objective respectively and $Z_{R}$ is the reference distance moved by the aspheric lens. The $512 \times 512$ images of the pollen grains at two separate focal planes, one at the origin where no remote focusing was used and the other with the remote focusing present, were taken at a frame rate of 15 frames per second using unidirectional scanning. No impairment from the AOD switching was observed on the alternating plane-to-plane scanning rate. 
(a)

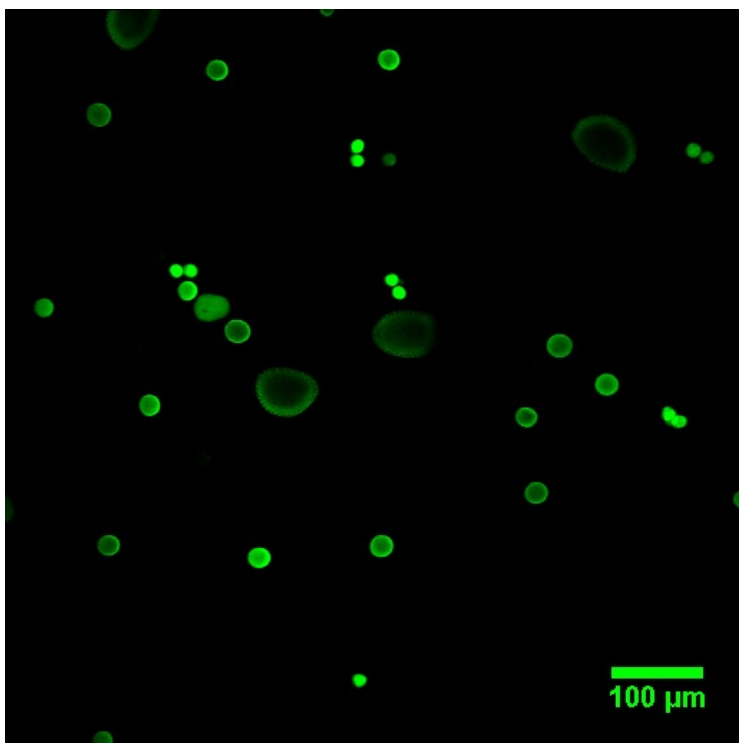

(b)

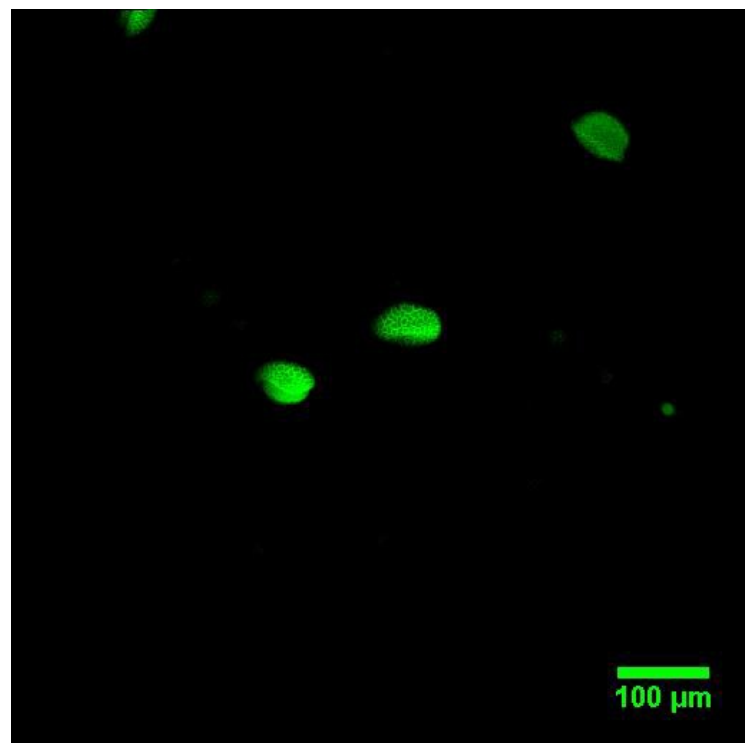

Figure 4. Autofluorescence images of pollen grains $2 \mathrm{P}$-excited at $\sim 35 \mathrm{~mW}, 900 \mathrm{~nm}$. (a) Pollen image at the origin (the beam path without remote focusing). (b) Pollen image with the focal plane upward shifted by $\sim 15 \mu \mathrm{m}$ via remote focusing.

Fig. 4 reveals clear differences in the structures of the pollen grains when the focal plane was axially moved away from the origin using the remote focusing.

\section{CONCLUSION}

We demonstrated the development and the performance of the fast multiplane 2P imaging system by combining an AOD and remote focusing, where the former provides the high optical switching rate and the latter offers the axial displacement of the focal spot without coming in contact with the objective or the specimen. Therefore, we anticipate the devised multiplane imaging technique will be useful in recording near-simultaneously the neuronal activities across separated planes within the volume of a mouse brain.

\section{ACKNOWLEDGMENTS}

The work is supported by UK Research Councils and Wellcome Trust. We acknowledge the software assistance from Jacob Franklin of Vidrio Technologies.

\section{REFERENCES}

[1] Denk, W., Strickler, J. and Webb, W., "Two-photon laser scanning fluorescence microscopy," Science 248, 73-76, (1990).

[2] Oheim, M., Beaurepaire, E., Chaigneau, E., Mertz, J. and Charpak, S., "Two-photon microscopy in brain tissue: parameters influencing the imaging depth," Journal of Neuroscience Methods 111, 29-37 (2001).

[3] Balu, M., Baldacchini, T., Carter, J., Krasieva, T. B., Zadoyan, R. and Tromberg, B. J., "Effect of excitation wavelength on penetration depth in nonlinear optical microscopy of turbid media," Journal of Biomedical Optics 14, 010508 (2009).

[4] Kobat, D., Horton, N. G. and Xu, C., "In vivo two-photon microscopy to 1.6-mm depth in mouse cortex," Journal of Biomedical Optics 16, 106014 (2011). 
[5] Kim, K. H., Buehler, C. and So, P. T., "High-speed, two-photon scanning microscope," Applied Optics 38, 60046009 (1999).

[6] Truong, T. V., Supatto, W., Koos, D. S., Choi, J. M. and Fraser, S. E., "Deep and fast live imaging with two-photon scanned light-sheet microscopy," Nature Methods 8, 757-760 (2011).

[7] Bewersdorf, J., Pick, R. and Hell, S. W., "Multifocal multiphoton microscopy," Optics Letters 23, 655-657 (1998).

[8] Zhu, G., Van Howe, J., Durst, M., Zipfel, W. and Xu, C., "Simultaneous spatial and temporal focusing of femtosecond pulses," Optics Express 13, 2153-2159 (2005).

[9] Nguyen, Q., Callamaras, N., Hsieh, C. and Parker, I., "Construction of a two-photon microscope for video-rate $\mathrm{Ca}^{2+}$ imaging," Cell Calcium 30, 383-393 (2001).

[10] Botcherby, E. J., Juskaitis, R., Booth, M. J. and Wilson, T., "Aberration-free optical refocusing in high numerical aperture microscopy," Optics Letters 32, 2007-2009 (2007).

[11] Dixon, R., "Acoustic diffraction of light in anisotropic media, " IEEE Journal of Quantum Electronics 3, 85-93 (1967).

[12] Zeng, S., Lv, X., Zhan, C., Chen, W. R., Xiong, W., Jacques, S. L. and Luo, Q., "Simultaneous compensation for spatial and temporal dispersion of acousto-optical deflectors for two-dimensional scanning with a single prism," Optics Letters 31, 1091-1093 (2006).

[13] Zeng, S., Lv, X., Bi, K., Zhan, C., Li, D., Chen, W. R., Xiong, W., Jacques, S. L. and Luo, Q., "Analysis of the dispersion compensation of acousto-optic deflectors used for multiphoton imaging," Journal of Biomedical Optics 12, 024015 (2007).

[14] Botcherby, E. J., Smith, C. W., Kohl, M. M., Débarre, D., Booth, M. J., Juškaitis, R., Paulsen, O. and Wilson, T., "Aberration-free three-dimensional multiphoton imaging of neuronal activity at $\mathrm{kHz}$ rates," Proceedings of the National Academy of Sciences 109, 2919-2924 (2012). 